\title{
Patterns of leukocyte recovery predict infectious complications after CD19 CAR-T cell therapy in a real-world setting
}

\author{
Astha Thakkar ${ }^{1 \#}$, Zhu Cui ${ }^{2 \#}$, Stephen Zachary Peeke ${ }^{3}$, Nishi Shah ${ }^{4}$, Kith Pradhan ${ }^{5}$, Amanda Lombardo ${ }^{1}$, \\ Fariha Khatun $^{1}$, Jennat Mustafa ${ }^{1}$, Alyssa De Castro ${ }^{1}$, Kailyn Gillick ${ }^{1}$, Felisha Joseph ${ }^{1}$, Anjali Naik ${ }^{1}$, Shafia \\ Rahman $^{1}$, Angelica D'Aiello ${ }^{2}$, Richard Elkind ${ }^{1}$, Susan Sakalian ${ }^{1}$, Karen Fehn ${ }^{1}$, Karen Wright ${ }^{1}$, \\ Michelly Abreu ${ }^{1}$, Latoya Townsend-Nugent ${ }^{1}$, Nicole Chambers ${ }^{1}$, Rosmi Mathew ${ }^{1}$, Donika Binakaj ${ }^{1}$, \\ Randin Nelson ${ }^{6}$, Carlo Palesi ${ }^{6}$, Monika Paroder ${ }^{6}$, Joan Uehlinger ${ }^{6}$, Yanhua Wang ${ }^{7}$, Yang Shi ${ }^{7}$, \\ Xingxing Zang ${ }^{5}$, Hao Wang ${ }^{5}$, Christopher Nishimura ${ }^{5}$, Xiaoxin Ren ${ }^{5}$, Ulrich G. Steidl ${ }^{8}$, Kira Gritsman ${ }^{1}$, \\ Murali Janakiram ${ }^{9}$, Noah Kornblum ${ }^{1}$, Olga Derman ${ }^{10}$, Ioannis Mantzaris ${ }^{1}$, Aditi Shastri ${ }^{1}$, Rachel Bartash ${ }^{11}$, \\ Yoram Puius $^{11}$, Margaret McCort ${ }^{11}$, Mendel Goldfinger ${ }^{1}$, Lizamarie Bachier-Rodriguez ${ }^{1}$, Amit Verma ${ }^{1}$, \\ Ira Braunschweig ${ }^{1}$, R. Alejandro Sica ${ }^{1}$
}

\footnotetext{
${ }^{1}$ Division of Oncology, Montefiore Medical Center/Albert Einstein College of Medicine, Bronx, NY, USA; ${ }^{2}$ Department of Internal Medicine, Montefiore Medical Center/Albert Einstein College of Medicine, Bronx, NY, USA; ${ }^{3}$ Department of Oncology, Maimonides Medical Center, Brooklyn, NY, USA; ${ }^{4}$ Memorial Sloan Kettering Cancer Center, New York, NY, USA; ${ }^{5}$ Albert Einstein College of Medicine, Bronx, NY, USA; ${ }^{6}$ Department of Transfusion Medicine, Montefiore Medical Center/Albert Einstein College of Medicine Bronx, NY, USA; ${ }^{7}$ Department of Pathology, Montefiore Medical Center/Albert Einstein College of Medicine Bronx, NY, USA; ${ }^{8}$ Department of Medicine, Albert Einstein College of Medicine Bronx, NY, USA; ${ }^{9}$ Department of Hematology, Oncology and Transplantation, University of Minnesota, Minneapolis, MN, USA; ${ }^{10}$ Boise VA Medical Center, Boise, ID, USA; ${ }^{11}$ Division of Infectious Diseases, Montefiore Medical Center/Albert Einstein College of Medicine, Bronx, NY, USA

Contributions: (I) Conception and design: SZ Peeke, N Shah, A Verma, RA Sica; (II) Administrative support: A Lombardo, F Khatun, J Mustafa, A De Castro, K Gillick, F Joseph, A Naik, S Rahman, A D'Aiello, R Elkind, S Sakalian, K Fehn, K Wright, M Abreu, L Townsend-Nugent, N Chambers, R Mathew, D Binakaj, R Nelson, C Palesi, M Paroder, J Uehlinger, Y Wang, Y Shi, X Zang, H Wang, C Nishimura, X Ren; (III) Provision of study materials or patients: None; (IV) Collection and assembly of data: A Thakkar, Z Cui, SZ Peeke; (V) Data analysis and interpretation: Z Cui, K Pradhan, A Thakkar, A Verma, RA Sica; (VI) Manuscript writing: All authors; (VII) Final approval of manuscript: All authors.

\#These authors contributed equally to this work.

Correspondence to: R. Alejandro Sica, MD. 111 East 210th Street, Bronx, NY 10467, USA. Email: asica@montefiore.org.
}

Background: Adoptive immunotherapy using CD19-targeted Chimeric antigen receptor T cells (CAR-T) has revolutionized the treatment of relapsed/refractory diffuse large B-cell lymphoma (DLBCL). Data is limited on the propensity of infections and lymphohematopoietic reconstitution after Day 30 (D30) following CAR-T cell therapy. In this study, we evaluated the prevalence and nature of infectious complications in an expanded cohort of DLBCL patients treated with CD19 CAR-T therapy and its association with the dynamics of leukocyte subpopulation reconstitution post-CAR-T cell therapy.

Methods: We conducted a retrospective study including 19 patients who received axicabtagene ciloleucel and investigated associations between cytopenia and infectious complications after D30.

Results: Nineteen patients were included, consisting of $42 \%$ Hispanic, 32\% Caucasian, 21\% AfricanAmerican, and 5\% Asian subjects. Post-D30 of CAR-T infusion, 47\% patients ( $\mathrm{n}=9)$ developed an infection and $53 \%(\mathrm{n}=10)$ remained infection-free. The most common infection type observed was viral (7 patients) followed by bacterial ( 5 patients) and fungal ( 3 patients). Of 25 total infectious events, $56 \%$ were grade 1 or 2 and 44\% were grade 3 with 10 being viral in etiology. To determine the kinetics of lymphohematopoietic reconstitution and its association with infection risk, we evaluated the relationship between cytopenias and rates of infection after D30. Notably, compared to non-infection group, infection group had a higher median absolute lymphocyte count (ALC) $(1,000 / \mu \mathrm{L}$ vs. $600 / \mu \mathrm{L}, \mathrm{P}<0.05)$, a lower median absolute neutrophil count (ANC)/ALC ratio (1.6 vs. 3.1, $\mathrm{P}<0.05)$ and a lower median AMC/ALC at D30 (0.37 vs. 1.67, $\mathrm{P}<0.05)$. In addition, we observed that only $22 \%$ of patients had recovered $\mathrm{ANC}>1,500 / \mu \mathrm{L}$ in the infection group as opposed to $70 \%$ in the non-infection group at D90 $(\mathrm{P}<0.05)$. Fifty-eight percent of the patients $(11 / 19)$ with 
relapsed refractory DLBCL achieved a complete response with a median follow-up of 233 days (7.7 months). Conclusions: Although CAR-T cell therapy is highly effective, infectious complications remain an important cause of morbidity and mortality. Low ANC/ALC and AMC/ALC ratios at D30 are potential novel predictors of infection and can be considered in future prophylactic strategies.

Keywords: Chimeric antigen receptor T cell (CAR-T) CD19 cells; infection; relapsed/refractory diffuse large B-cell lymphoma (DLBCL); axicabtagene ciloleucel; real-world cohort

Received: 25 January 2021; Accepted: 03 June 2021; Published: 06 September 2021. doi: 10.21037/sci-2021-008

View this article at: https://dx.doi.org/10.21037/sci-2021-008

\section{Introduction}

Chimeric antigen receptor $\mathrm{T}$ cell (CAR-T) therapy targeted against CD19 has emerged as a new treatment for relapsed/refractory B cell malignancies, including acute lymphoblastic leukemia (ALL), non-Hodgkin's lymphoma (NHL) and chronic lymphocytic leukemia (CLL) $(1,2)$. Prior to receiving CAR-T cells, patients receive lymphodepleting chemotherapy, typically consisting of fludarabine and cyclophosphamide (3); such therapy typically leads to cytopenia and may impair host immune defenses, leading to infectious complications. Moreover, targeting CD19 B cells leads to lymphodepletion and may cause hypogammaglobulinemia $(4,5)$. Thus, it is important to assess the rates of infectious complications post CAR-T cell therapy, especially in real-world settings.

As more CAR-T cell therapies become available for different malignancies, it is becoming increasingly important to recognize and manage early and late toxicities. The two major early toxicities of CAR-T cell therapy that have been identified include cytokine release syndrome (CRS) and immune effector cell-associated neurotoxicity syndrome (ICANS) (6). As patients receiving CAR-T cell therapies are followed longitudinally, a number of studies evaluating delayed toxicities, including hematopoietic system-related adverse events and infections, as well as the association between various clinical parameters such as CRS, and infectious complications in these patients have been published recently $(1,5,7)$. These studies have indicated that most infectious complications occur in the first 30 days after CAR-T cell infusion $(8,9)$. Most of the data have been obtained from clinical trials, and it is important to evaluate the complication rates in a real-world setting. Thus, we aimed to study the rates of hematologic reconstitution and the rate of cytopenia, including prolonged cytopenia (after 90 days), induced by CAR-T cell infusion in an ethnically diverse patient population and to monitor the occurrence of infectious complications beyond day 30 (D30). Furthermore, we aimed to study the association between the ratios of various leukocyte subsets at D30 and infection. We present the following article in accordance with the STROBE reporting checklist (available at https://dx.doi.org/10.21037/sci-2021-008).

\section{Methods}

We conducted a retrospective review of 19 adult patients with relapsed/refractory diffuse large B-cell lymphoma (DLBCL) treated with CD19-directed CAR-T cell therapy at Montefiore Medical Center from 2018 to 2020. Demographic variables collected included age, sex and ethnicity. Data pertaining to initial diagnosis, features of the malignancy, treatment history, prior autologous stem cell transplantation, timing of leukapheresis and the infusion of CAR-T cells, and absolute neutrophil count (ANC), absolute lymphocyte count (ALC) and absolute monocyte count (AMC) following CAR-T cell infusion at the D30, D30-90, D90-180 and D180-365 time points were also collected. To assess early hematologic recovery, we also recorded the maximum and minimum values between D20-40 for ANC, ALC and AMC. To study hematologic recovery at D90, we recorded the ANC, ALC, and AMC values available between D70 and D110. Data regarding fever, grade of CRS, neurotoxicity and levels of $\mathrm{C}$-reactive protein (CRP) were also collected during index admission. We also recorded the use of tocilizumab and steroids for the management of CRS and neurotoxicity, respectively. Patients received levofloxacin, valacyclovir and fluconazole for infectious prophylaxis after CAR-T therapy until ANC >500 per institutional guidelines. The study was conducted in accordance with the Declaration 
Table 1 Baseline characteristics of patients receiving CD19 CAR-T cell therapy

\begin{tabular}{|c|c|}
\hline Characteristic & Value \\
\hline Median age, years & $61[49-84]$ \\
\hline \multicolumn{2}{|l|}{ Sex, $\mathrm{n}[\%]$} \\
\hline Male & 10 [53] \\
\hline Female & $9[47]$ \\
\hline \multicolumn{2}{|l|}{ Race, n [\%] } \\
\hline Hispanic & 8 [42] \\
\hline White & $6[32]$ \\
\hline African American & $4[21]$ \\
\hline Asian & $1[5]$ \\
\hline \multicolumn{2}{|l|}{ Lymphoma subtype, n [\%] } \\
\hline Primary DLBCL & 14 [74] \\
\hline Prior follicular lymphoma & $3[16]$ \\
\hline Prior SLL/CLL/LPL & $1[5]$ \\
\hline HIV-associated lymphoma & $1[5]$ \\
\hline Germinal center lymphoma & 9 [47] \\
\hline Double-/triple-hit lymphoma & $2[10]$ \\
\hline Double-triple-expressor lymphoma & 15 [79] \\
\hline Central nervous system involvement & $2[11]$ \\
\hline Median lines of prior chemotherapy & $3[1-9]$ \\
\hline Prior autologous stem cell transplant & $9[47]$ \\
\hline Chronic hepatitis B infection & $3[16]$ \\
\hline
\end{tabular}

CAR-T, Chimeric antigen receptor $\mathrm{T}$ cell.

of Helsinki (as revised in 2013). The study was approved by ethics board of Albert Einstein College of Medicine (NO.: IRB 2014-3056) and informed consent was waived for individual participants.

\section{Statistical analysis}

Associations between the presence of infection and D30 ANC, ALC, AMC, the ANC/ALC ratio, and the AMC/ALC ratio were assessed using the Kruskal-Wallis test. Associations between infection and hematologic reconstitution at D90 were determined using the chi square test. Kaplan-Meier curves and the log-rank test were used to evaluate overall survival (OS) and progression-free survival (PFS).

\section{Definition of infection}

Infection was noted if the patient met the relevant clinical and/or microbiologic criteria. Clinical criteria included symptoms or radiographic findings suggestive of an infection. Microbiologic criteria included positive blood cultures, urine cultures, nasopharyngeal/oropharyngeal viral swabs, serum tests for fungal infection or tests for SARS$\mathrm{CoV}-2$. Patients with human immunodeficiency virus (HIV) were not excluded. Infections were graded using the BMT CTN Severity Grading Table and Recurrence Interval Definitions.

\section{Definition of hematologic recovery}

An ANC of $>1,500 / \mu \mathrm{L}$ at day 90 post CAR-T cell infusion was defined as hematologic recovery. For patients who did not have an exact D90 ANC value, the highest value between D70 and D110 was chosen.

\section{Results}

\section{The study population was an ethnically diverse inner-city population with relapsed DLBCL}

A total of 19 patients were included in our study. The study population comprised ten (53\%) males and 9 (47\%) females. The median age at the time of CAR-T cell therapy was 63 years. Forty-two percent of patients were Hispanic, 32\% were Caucasian, 21\% were African American and $5 \%$ were Asian. The majority of patients, 14 out of 19 (74\%), had primary relapsed/refractory DLBCL. Sixteen percent of patients (3/19) had prior follicular lymphoma that transformed into DLBCL. One patient had prior lymphoplasmacytic lymphoma that transformed into DLBCL. One patient with DLBCL had concomitant infection with HIV, three patients had chronic hepatitis B and two patients had CNS involvement of lymphoma prior to CAR-T cell therapy. Overall, patients had received a median of three lines of therapy prior to CAR-T cell therapy. Nine patients (47\%) had received high-dose chemotherapy with autologous stem cell transplant rescue prior to CAR-T cell therapy. The baseline characteristics of our cohort are summarized in Table 1.

\section{There was a high rate of clinical CR seen with CD19 CAR-Ts}

Fifty-eight percent of the patients (11/19) with relapsed 


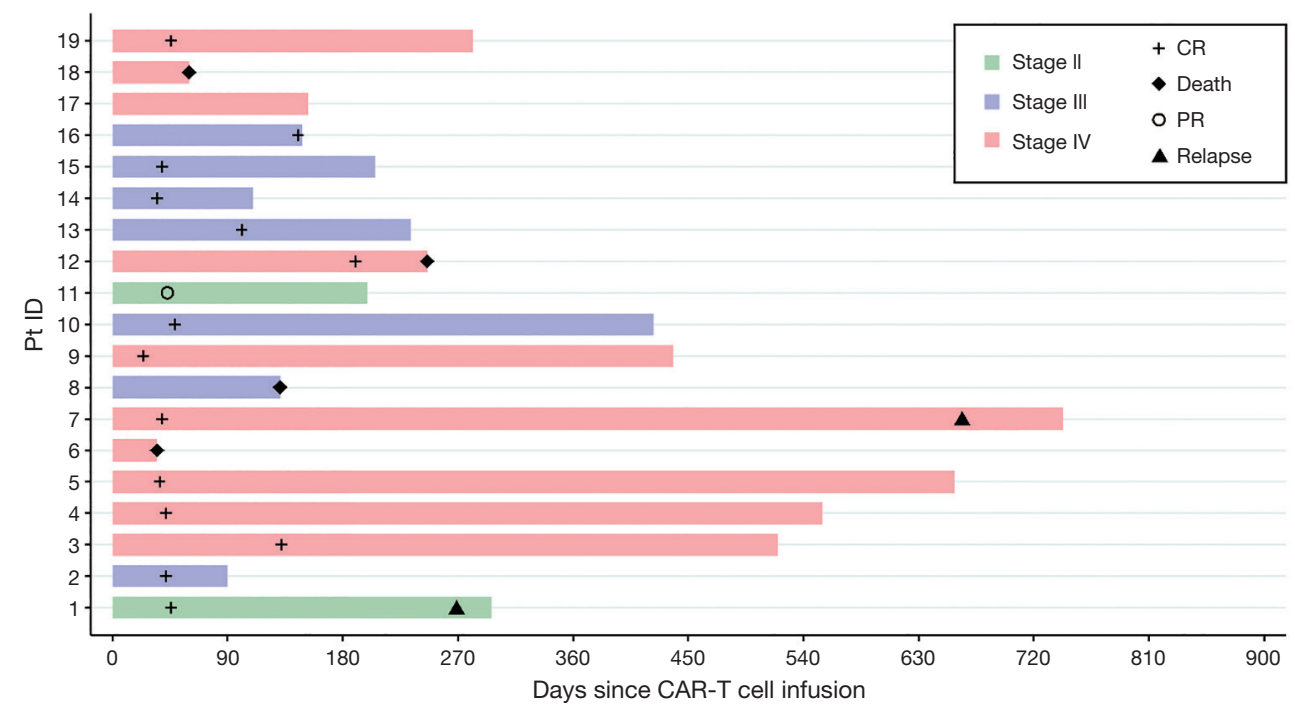

Figure 1 Clinical outcomes for patients with NHL post CD19 CAR-T cell therapy. Swimmer's plot showing 8/13 patients in CR at 1 year. CAR-T, Chimeric antigen receptor T cell; NHL, non-Hodgkin's lymphoma.

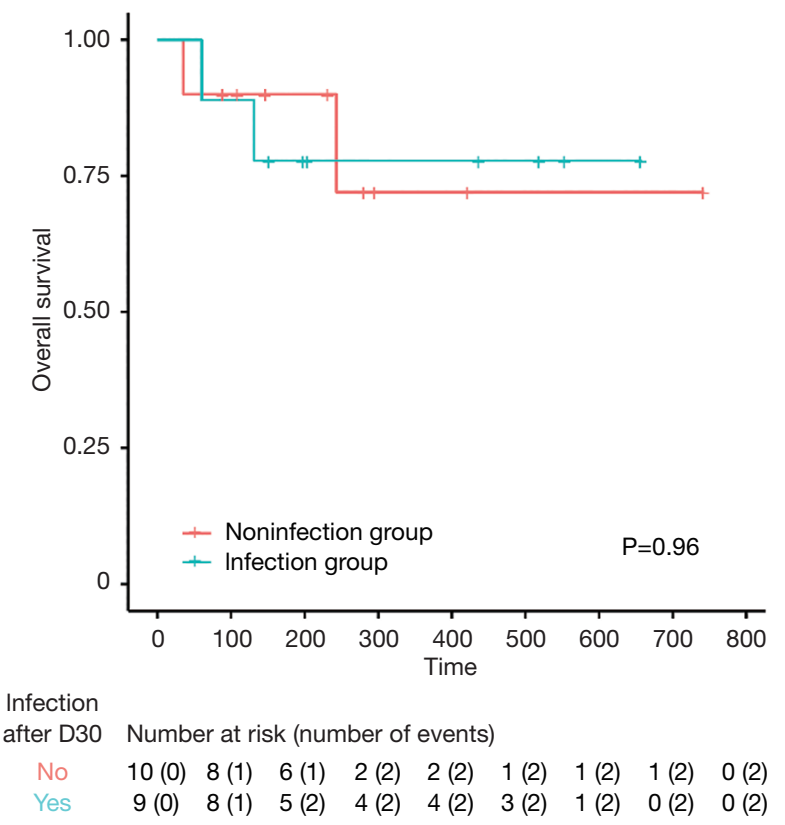

Figure 2 Overall survival.

refractory DLBCL achieved a complete response (CR) with a median follow-up of 233 days ( 7.7 months). Sixtytwo percent (8/13) of evaluable patients maintained CR at one year of follow-up (Figure 1). The median OS was not reached in our population (Figure 2). Four patients out of the entire cohort died. The cause of death in three patients was progression of disease, and in one patient, the cause of death was presumed to be respiratory infection (event at outside hospital). The median PFS was also not reached in our population (Figure 3). For PFS analysis, we excluded two patients who died during the initial CAR-T cell admission because there was no time point to assess response to therapy

\section{The majority of infections were grade 1 or 2 and viral in etiology}

Infectious complications beyond D30 occurred in 9 out of 19 patients. This represents an infection rate of $47 \%$ in our patient population. A total of 25 infectious events were recorded beyond D30. The majority of the infections were viral. Viral infections were identified in 7/19 (37\%) patients. The next most common infectious events were bacterial infections [which occurred in $5 / 19$ patients $(26 \%)$ ] and fungal infections [which occurred in 3/19 patients (16\%)]. The specific organisms causing the infections and the grade of infection severity in the patients are summarized in Table 2.

Bacterial agents included infection with Clostridium difficile, Escherichia coli and Pseudomonas aeruginosa. One patient with chronic HIV infection developed disseminated cryptococcosis with multiple episodes of cryptococcal meningitis. Infections were graded according to the BMT CTN Infection Severity Grading Table and Recurrence Interval Definitions (version 3.0, dated March 19, 2013). 


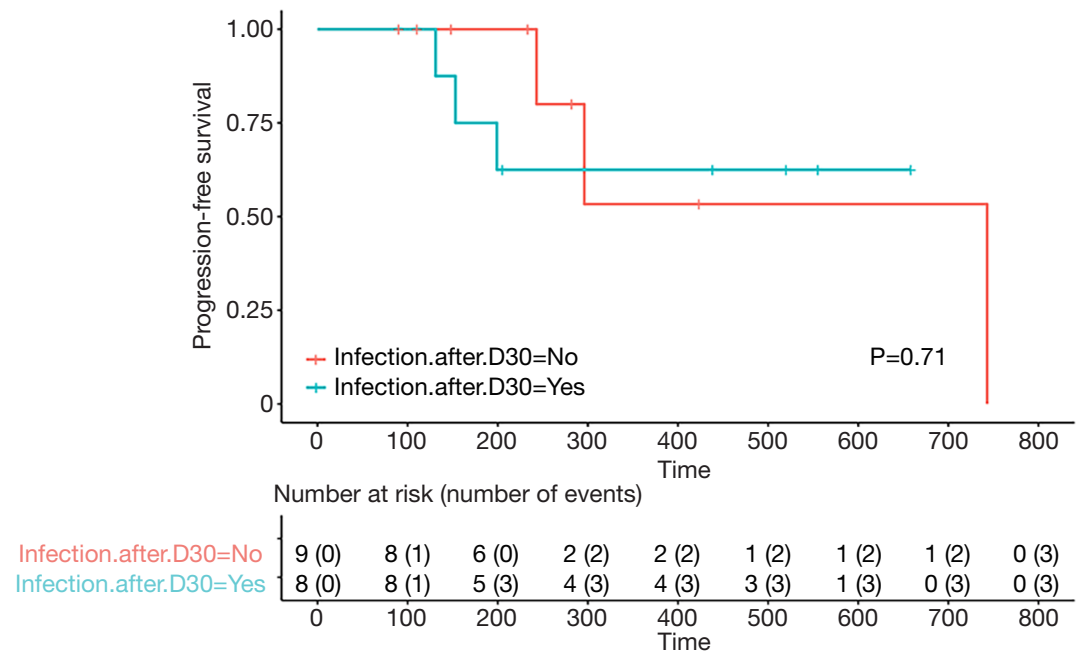

Figure 3 Progression-free survival.

Table 2 Type and grade of infection post CD19 CAR-T cell therapy in NHL

\begin{tabular}{|c|c|c|c|c|c|c|}
\hline $\begin{array}{l}\text { Patients with } \\
\text { infection }\end{array}$ & Bacteria & Virus & Fungus & D30 ANC & D30 ALC & D30 AMC \\
\hline 1 & & Influenza A (G3) & & 1.1 & 0.2 & 0.35 \\
\hline 3 & Escherichia coli UTI (G1) & SARS-CoV-2 (G3) & & 0.7 & 0.5 & 0.2 \\
\hline 5 & $\begin{array}{l}\text { Pseudomonas pneumonia } \\
\text { (G2), Pseudomonas } \\
\text { bacteremia (G3), } \\
\text { pneumonia NOI (G2) }\end{array}$ & $\begin{array}{l}\text { Influenza A (G3), } \\
\text { Parainfluenza (G3), CMV } \\
\text { viremia (G2), SARS-CoV-2 } \\
\text { (G3), BK viruria (G1), HSV } \\
\text { keratitis (G2) }\end{array}$ & $\begin{array}{l}\text { Cryptococcal meningitis } \\
\text { (G3), disseminated } \\
\text { cryptococcosis (G3) }\end{array}$ & 0.5 & 0.5 & 0.1 \\
\hline 7 & & SARS-CoV-2 (G3) & & 0.8 & 1.9 & 0.4 \\
\hline 8 & Clostridium difficile (G2) & CMV viremia (G2) & & 0.5 & 0.9 & 0.32 \\
\hline 9 & $\begin{array}{l}\text { Vancomycin-resistant } \\
\text { enterococcus UTI (G1) }\end{array}$ & Rhinovirus (G2), CMV (G1) & & 2.3 & 0.1 & 0.3 \\
\hline
\end{tabular}

*, Test results post-mortem. Infections were graded using the BMT CTN Severity Grading Table and Recurrence Interval Definitions. Five patients had bacterial infections, seven patients had viral infections, and three patients had fungal infections. In terms of severity, five infectious events were grade 1, nine were grade 2, and eleven were grade 3. CAR-T, Chimeric antigen receptor T cell; NHL, non-Hodgkin's lymphoma; ANC, absolute neutrophil count; ALC, absolute lymphocyte count; AMC, absolute monocyte count.

Out of a total of 25 infection events, we found that $14 / 25$ (56\%) were grade 1 or 2 , and $11 / 25(44 \%)$ were grade 3 in severity. Both patients presented with respiratory failure with imaging findings suggesting pneumonia. One of these patients died at an outside hospital. The patient was suspected to have a respiratory infection versus progression 
Table 3 Clinical variables associated with infections post CD19 CAR-T cell therapy for NHL

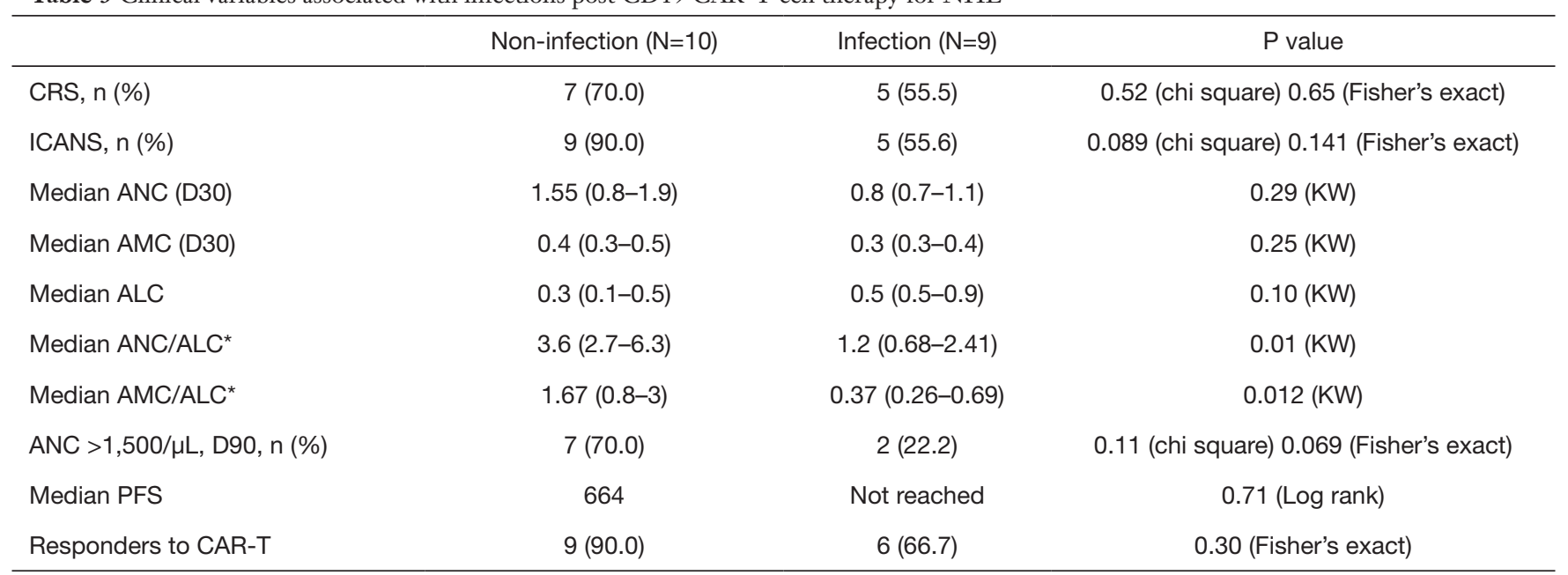

The ANC, AMC, and ALC values at D30 were calculated using minimum and maximum values between D20 to D40. ${ }^{*}$ The infection group had 8 people, and the non-infection group had 9 people because some patients had 0 as a minimum ALC level. CAR-T, Chimeric antigen receptor T cell; NHL, non-Hodgkin's lymphoma; ANC, absolute neutrophil count; ALC, absolute lymphocyte count; AMC, absolute monocyte count; KW, Kruskal Wallis.

of disease; however, the details of the full hospital course were not available. Two patients had cytomegalovirus (CMV) reactivation and viremia requiring antiviral therapy. The infection timings are summarized in Table S1.

\section{Patients with COVID-19 infections recovered without sequelae}

All three patients who developed infections with confirmed SARS-CoV-2 positivity recovered. The COVID infection was noted at D150, 389 and 630 for these patients. Two patients received inpatient care within Montefiore. One of the patients received tocilizumab and was enrolled in a convalescent plasma clinical trial. The patient showed signs of clinical improvement and was discharged on hospital D12. Notably, he developed profound lymphopenia during his hospital stay for COVID-19 and had an ALC ranging from 100 to $300 / \mu \mathrm{L}$ at D150-161 post CAR-T cell therapy. The second patient with COVID-19 had multiple comorbidities, including a well-controlled chronic HIV infection and IgG subclass deficiency. This patient received remdesivir and steroids for COVID-19 and was also enrolled in a convalescent plasma clinical trial. The patient recovered from COVID-19 and was discharged at hospital D14. However, he had prolonged shedding of SARS-CoV-2, and a positive PCR was noted 50 days after the initial infection, as of the cut-off date for our study.
The third patient received care at an outside hospital and presented for follow-up after recovering from COVID-19.

\section{Lower ANC/ALC and AMC/ALC ratios between D20 and $D 40$ were associated with infection}

To determine laboratory biomarkers predictive of infectious complications, we next evaluated cytopenia post therapy. The cohort of patients without any infections (non-infection group, $n=10$ ) was compared to the cohort of patients who developed any infection (infection group, $n=9$ ). We observed that the infection group had a higher median maximum ALC than the non-infection group $(1,000 / \mu \mathrm{L} v s$. $600 / \mu \mathrm{L}, \mathrm{P}=0.034)$. The median minimum $\mathrm{ANC}$ was found to be lower in the infection group than the non-infection group, even though statistical significance was not reached $(800 / \mu \mathrm{L}$ vs. $1,550 / \mu \mathrm{L}, \mathrm{P}=0.29)$. The median maximum and minimum AMCs were similar in both groups. Furthermore, we observed that the median minimum ANC/ALC ratio and median minimum AMC/ALC ratios were significantly lower in the cohort of patients who developed infection than the cohort of patients who did not develop infection. In the infection group, the median minimum ANC/ALC ratio was lower than that in the non-infection group (1.2 vs. 3.6, $\mathrm{P}=0.01)$. The median minimum $\mathrm{AMC} / \mathrm{ALC}$ ratio was lower in the infection group than in the non-infection group at $\mathrm{D} 30$ ( 0.37 vs. $1.67, \mathrm{P}=0.01)$. 
In addition, we also observed that at $\mathrm{D} 90$, the infection group had a lower rate of hematologic reconstitution (ANC $>1,500 / \mu \mathrm{L}$ ) than the non-infection group. We observed that only $22 \%(\mathrm{n}=2)$ of patients had recovered $\mathrm{ANC}>1,500 / \mu \mathrm{L}$ in the infection group while $70 \%(n=7)$ had recovered ANC $>1,500 / \mu \mathrm{L}$ in the non-infection group at $\mathrm{D} 90(\mathrm{P}=0.11)$. These findings suggest that a trend toward incomplete hematologic reconstitution at D90 may be associated with an increased propensity for infection. These findings are summarized in Table 3.

We also recorded the frequencies of prolonged cytopenia after CAR-T cell therapy. These findings along with the severities of the cytopenia are summarized in Table S2.

\section{CRS and ICANS}

The rates of CRS were comparable between the two groups (55.6\% and $70 \%$ in the infection and non-infection groups, respectively, $\mathrm{P}=0.52$ ). Surprisingly, the rate of ICANS was lower $(55.6 \%)$ in the infection group than in the noninfection group (90\%), although not statistically significant $(\mathrm{P}=0.09)$. A total of twelve patients experienced CRS, of whom 8 experienced grade $1 \mathrm{CRS}$ and 4 experienced grade 2-3 CRS. Tocilizumab was used to treat CRS in six patients. ICANS was noted in 14 patients, of whom 11 patients had grade 1-2 ICANS. Three patients had grade 3-4 events necessitating the use of corticosteroids. These data are summarized in Table S3.

\section{Discussion}

CD 19-targeted CAR-T cell adoptive immunotherapy is a newly available treatment option for patients with relapsed/ refractory B cell malignancies, including B cell ALL and DLBCL, and it has shown high efficacy rates in several phase 1 and 2 trials $(6,10,11)$. The main early toxicities described for CAR-T cell therapy so far are CRS and ICANS. The combination of CD-19-targeted T cells with the lymphodepleting conditioning regimens that patients receive prior to CAR-T cell infusion leads to cytopenia and an increased likelihood of infection in patients. The data on long-term cytopenia and infections in this patient population are still being collected.

The first clinical trials evaluating the use of CAR-T cell therapy, including trials employing tisagenlecleucel (tisa-cel, Kymriah) and, more recently, axicabtagene ciloleucel (Axi-cel, Yescarta), have reported frequencies of delayed neutropenia, anemia and thrombocytopenia at D90 ranging from $11 \%$ to $20 \%(2,8,11)$.

A recent retrospective analysis studying cytopenia in patients treated with CAR-T cell therapy reported that while the majority of patients had hematologic recovery $(\mathrm{Hb}$ $>8 \mathrm{~g} / \mathrm{dL}, \mathrm{WBC}>3,000 / \mu \mathrm{L}$ ANC $>1,000 / \mu \mathrm{L}$ for two weeks without transfusion or growth factor support, and platelets $>50,000 / \mu \mathrm{L}$ for 1 week without transfusion support) within the first three months post CAR-T cell therapy, complete normalization of counts was observed in only $15 \%$ patients by 3 months and in only $60 \%$ of patients by 9 months following the infusion. This study included all CAR-T cell products, including investigational anti-BCMA CAR-T cell therapy (12).

Furthermore, a retrospective study by Fried et al. (13) noted that patients undergoing CAR-T cell therapy may experience cytopenia in a biphasic manner. Specifically, the study suggested that delayed cytopenia, defined in that study as those cytopenia occurring beyond D21, may be directly related to the infused CAR-T cells and unrelated to the initial lymphodepleting therapy as well as the potential myelosuppression induced by CRS (13).

It is not surprising that prolonged neutropenia can predispose patients to infectious complications. In the updated analysis of the pivotal phase 2 multicenter ZUMA1 trial, 9 infectious events were reported in 8 patients. The majority of these events were viral or bacterial respiratory tract infections (6). The longest follow-up in this report was 18.6 months. A retrospective study by Hill and colleagues analyzed 133 patients with relapsed/ refractory B cell malignancies receiving CAR-T cell therapy for infectious complications. This study reported that 23 infectious events occurred in 17/119 (14\%) patients between 29 and 90 days after CAR-T cell infusion. A study also reported that patients who had received $>4$ prior lines of chemoimmunotherapy and patients receiving a higher CAR-T cell dose had an increased risk of infection (1).

A recent retrospective analysis by Logue et al. (7) studied the association between hematopoietic recovery and infection. They reported an infection rate of $44.3 \%$ after D30 in a cohort of seventy patients. The study reported that most infections were non severe. There was no association between low CD4 and CD8 counts at D30 and infection after D30 (7).

In this paper, we described the clinical characteristics and infectious complications beyond D30 in a multiethnic underserved patient population from New York City. This was a significantly pretreated population with a median of three prior lines of therapy. Forty-seven percent of patients 
had received an autologous stem cell transplantation prior to CAR-T cell therapy. The majority of patients (53\%) remained infection-free beyond D30 after CAR-T cell therapy. Among the patients who developed infections, most patients had respiratory infections. This finding is similar to what has been reported in prior studies $(1,9)$. Viral agents included Rhinovirus, Influenza A virus, and Parainfluenza virus, and three patients had infections with SARS-CoV-2. The two patients treated at Montefiore Medical Center for COVID-19 recovered from the infection. One patient was enrolled in a convalescent plasma clinical trial as well. Notably, this patient recovered their counts post CAR-T cell therapy; however, during infection with SARS-CoV-2, the patient experienced profound lymphopenia (ALC 100 $300 / \mu \mathrm{L}$ ). This lymphopenia occurred from D151 to D160 post CAR-T cell therapy. The second patient with SARS$\mathrm{CoV}-2$ infection had coinfection with HIV and an IgG subclass deficiency. This patient also received remdesivir and was enrolled in a convalescent plasma trial. While his clinical status had stabilized, his SARS-CoV-2 PCR result remained positive after 50 days. No further testing results available before the end study follow-up. This patient also harbored chronic CMV and cryptococcal infections and has had multiple hospitalizations for cryptococcal meningitis as well as multiple episodes of multidrug-resistant bacterial infections given his underlying immunodeficiency. It has been one year and 3 months since his CAR-T cell therapy, and his lymphoma continues to remain in remission.

One significant finding of our study is that the maximum ALC early after CAR-T cell therapy (D20-40) may be associated with delayed infectious complications. In addition, the finding that low minimum ANC/ALC and low minimum AMC/ALC ratios at D30 were seen in the group that had infections may suggest that the presence of slow/prolonged neutrophil and monocyte recovery can help identify patients who may benefit from increased surveillance and close clinical follow-up. In addition, an ANC $<1,500 / \mu \mathrm{L}$ at D90 may also help identify patients who would be at increased risk of delayed infectious complications.

Given that CD19 is the target of CAR-T cell therapies for B cell malignancies, B cell aplasia has been reported in many studies, and it can cause low immunoglobulin levels sometimes up to 6 months post CAR-T cell therapy. In our cohort, while immunoglobulin levels were not obtainable for all patients, one patient who received serial assessment of immunoglobulin levels remained infectionfree throughout her course and follow-up (her IgG levels have remained less than $400 \mathrm{mg} / \mathrm{dL}$ for over one year). This finding adds to existing observations that some degree of humoral immunity is preserved despite CAR-T cell therapy $(2,14,15)$.

The median OS was not reached in our group. This is likely due to the small sample size. The median PFS in the group without infection was 22.1 months but was not reached in the group with infection $(\mathrm{P}=0.71)$. Infection due to an unknown respiratory infection in the midst of the COVID-19 pandemic (at the time NYC was the epicenter) was possibly a contributor of death in one out of the 19 patients $(5.2 \%)$ in our cohort. Of note, due to the small number of patients and the absence of COVID-19 related deaths in the other cohorts it is hard to make comparisons with other real world cohorts at this time $(1,7,16)$. Despite prolonged cytopenia and B cell aplasia, most patients in our cohort were able to recover from infection events.

The limitations of our study include its retrospective design and small sample size.

In summary, our analysis suggests that the D30 ANC/ALC ratio and AMC/ALC ratio may be helpful for identifying patients who are likely to develop infectious complications post CAR-T cell therapy. These patients may benefit from increased infectious disease surveillance and potentially longer antibiotic prophylaxis. Larger, prospective studies are needed to validate these findings.

\section{Acknowledgments}

We would like to thank all Northwest 2 nurses (MMC), outpatient infusion nurses and pharmacists involved in the care of the patients involved in this study.

Funding: None.

\section{Footnote}

Reporting Checklist: The authors have completed the STROBE reporting checklist. Available at https://dx.doi. org/10.21037/sci-2021-008

Conflicts of Interest: All authors have completed the ICMJE uniform disclosure form (available at https://dx.doi. org/10.21037/sci-2021-008). Dr. Verma serves as an unpaid editorial board member of Stem Cell Investigation. Dr. Steidl has received research funding from GlaxoSmithKline, Bayer Healthcare, Aileron Therapeutics, Novartis, has received compensation for consultancy services and for serving on 
scientific advisory boards from GlaxoSmithKline, Bayer Healthcare, Novartis, Celgene, Aileron Therapeutics, Stelexis Therapeutics, Pieris Pharmaceuticals, Vor Biopharma, and Trillium Therapeutics, and has equity ownership in and is serving on the board of directors of Stelexis Therapeutics. Dr. Gritsman has received Research funding from iOnctura, SA. Dr. Shastri has received research funding from Kymera Therapeutics, consultancy fees from Guidepoint \& GLG, honoraria from OncLive. Dr. Verma has received research funding from GlaxoSmithKline, BMS, Jannsen, Incyte, MedPacto, Celgene, Novartis, Curis, Prelude and Eli Lilly and Company, has received compensation as a scientific advisor to Novartis, Stelexis Therapeutics, Acceleron Pharma, and Celgene, and has equity ownership in Throws Exception and Stelexis Therapeutics. The other authors have no conflicts of interest to declare.

Ethical Statement: The authors are accountable for all aspects of the work in ensuring that questions related to the accuracy or integrity of any part of the work are appropriately investigated and resolved. The study was conducted in accordance with the Declaration of Helsinki (as revised in 2013). The study was approved by ethics board of Albert Einstein College of Medicine (NO.: IRB 2014-3056) and informed consent was waived for individual participants.

Open Access Statement: This is an Open Access article distributed in accordance with the Creative Commons Attribution-NonCommercial-NoDerivs 4.0 International License (CC BY-NC-ND 4.0), which permits the noncommercial replication and distribution of the article with the strict proviso that no changes or edits are made and the original work is properly cited (including links to both the formal publication through the relevant DOI and the license). See: https://creativecommons.org/licenses/by-nc-nd/4.0/.

\section{References}

1. Hill JA, Li D, Hay KA, et al. Infectious complications of CD19-targeted chimeric antigen receptor-modified T-cell immunotherapy. Blood 2018;131:121-30.

2. Locke FL, Ghobadi A, Jacobson CA, et al. Longterm safety and activity of axicabtagene ciloleucel in refractory large B-cell lymphoma (ZUMA-1): a single-arm, multicentre, phase 1-2 trial. Lancet Oncol
2019;20:31-42.

3. Park JH, Geyer MB, Brentjens RJ. CD19-targeted CAR T-cell therapeutics for hematologic malignancies: interpreting clinical outcomes to date. Blood 2016;127:3312-20.

4. Hill JA, Giralt S, Torgerson TR, et al. CAR-T - and a side order of $\mathrm{IgG}$, to go? - Immunoglobulin replacement in patients receiving CAR-T cell therapy. Blood Rev 2019;38:100596.

5. Vora SB, Waghmare A, Englund JA, et al. Infectious complications following CD19 chimeric antigen receptor T-cell therapy for children, adolescents, and young adults. Open Forum Infect Dis 2020;7:ofaa121.

6. Neelapu SS, Locke FL, Bartlett NL, et al. Axicabtagene ciloleucel CAR T-cell therapy in refractory large B-cell lymphoma. N Engl J Med 2017;377:2531-44.

7. Logue JM, Zucchetti E, Bachmeier CA, et al, et al. Immune reconstitution and associated infections following axicabtagene ciloleucel in relapsed or refractory large B-cell lymphoma. Haematologica 2021;106:978-86.

8. Cordeiro A, Bezerra ED, Hirayama AV, et al. Late events after treatment with CD19-targeted chimeric antigen receptor modified T cells. Biol Blood Marrow Transplant 2020;26:26-33.

9. Wudhikarn K, Palomba ML, Pennisi M, et al. Infection during the first year in patients treated with CD19 CAR T cells for diffuse large B cell lymphoma. Blood Cancer J 2020;10:79.

10. Schwartz JD. Tisagenlecleucel in diffuse large B-cell lymphoma. N Engl J Med 2019;380:1585-6.

11. Maude SL, Laetsch TW, Buechner J, et al. Tisagenlecleucel in children and young adults with B-cell lymphoblastic leukemia. N Engl J Med 2018;378:439-48.

12. Jain T, Knezevic A, Pennisi M, et al. Hematopoietic recovery in patients receiving chimeric antigen receptor T-cell therapy for hematologic malignancies. Blood Adv 2020;4:3776-87.

13. Fried S, Avigdor A, Bielorai B, et al. Early and late hematologic toxicity following CD19 CAR-T cells. Bone Marrow Transplant 2019;54:1643-50.

14. Kochenderfer JN, Somerville RPT, Lu T, et al. Longduration complete remissions of diffuse large B cell lymphoma after anti-CD19 chimeric antigen receptor $\mathrm{T}$ cell therapy. Mol Ther 2017;25:2245-53.

15. Schuster SJ, Svoboda J, Chong EA, et al. Chimeric antigen receptor $\mathrm{T}$ cells in refractory B-cell lymphomas. $\mathrm{N}$ Engl J 
Med 2017;377:2545-54.

16. Wudhikarn K, Pennisi M, Recio MG, et al. Infectious complications in aggressive B cell non-hodgkin lymphoma after CD-19 chimeric antigen receptor T cell therapy. Biol Blood Marrow Transplant 2020;26:S326.

doi: $10.21037 /$ sci-2021-008

Cite this article as: Thakkar A, Cui Z, Peeke SZ, Shah N, Pradhan K, Lombardo A, Khatun F, Mustafa J, De Castro A, Gillick K, Joseph F, Naik A, Rahman S, D'Aiello A, Elkind R, Sakalian S, Fehn K, Wright K, Abreu M, TownsendNugent L, Chambers N, Mathew R, Binakaj D, Nelson R, Palesi C, Paroder M, Uehlinger J, Wang Y, Shi Y, Zang X, Wang H, Nishimura C, Ren X, Steidl UG, Gritsman K, Janakiram M, Kornblum N, Derman O, Mantzaris I, Shastri A, Bartash R, Puius Y, McCort M, Goldfinger M, BachierRodriguez L, Verma A, Braunschweig I, Sica RA. Patterns of leukocyte recovery predict infectious complications after CD19 CAR-T cell therapy in a real-world setting. Stem Cell Investig 2021;8:18. 


\section{Supplementary}

Table S1 Types and timing of infections

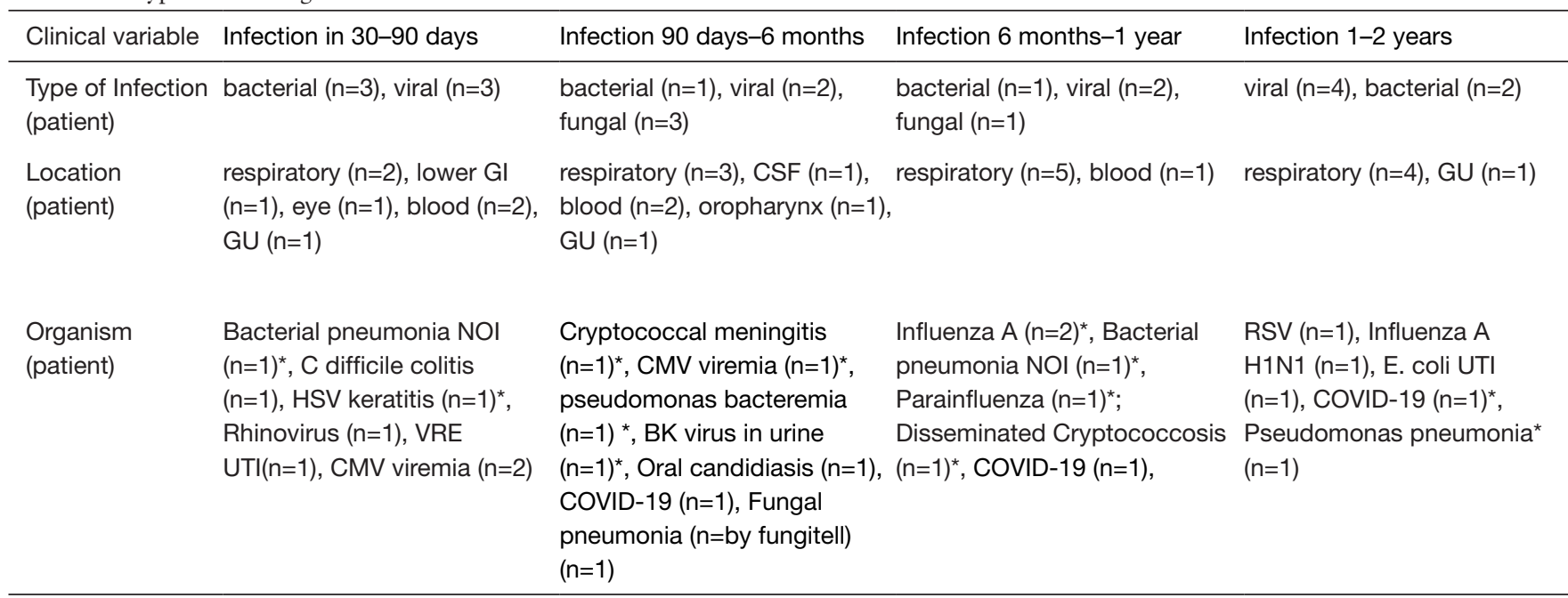

*, Patient with HIV on ART. GU, genitourinary; CSF, cerebrospinal fluid; NOI, no organism identified; C. difficile, clostridium difficile; VRE, vancomycin-resistant enterococcus; UTI, urinary tract infection; CMV, cytomegalovirus; COVID-19, coronavirus disease 19; RSV, respiratory syncytial virus

Table S2 Prevalence of cytopenia after CAR-T cell therapy.

\begin{tabular}{|c|c|c|c|c|}
\hline Cytopenia & D30-90 (n=19) & D90-180 (n=14v & D180-1 year $(n=11)$ & $1-2$ years $(n=5)$ \\
\hline \multicolumn{5}{|l|}{ Neutropenia, n [\%] } \\
\hline Normal $(\geq 1,500 / \mu \mathrm{L})$ & $5[26]$ & $7[50]$ & 6 [55] & $1[20]$ \\
\hline 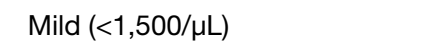 & $5[26]$ & $1[7]$ & $4[36]$ & $1[20]$ \\
\hline Moderate $(<1,000 / \mu \mathrm{L})$ & $8[42]$ & $5[36]$ & $0[0]$ & $1[20]$ \\
\hline Severe $(<500 / \mu \mathrm{L})$ & $1[5]$ & $1[7]$ & $1[9]$ & $2[40]$ \\
\hline \multicolumn{5}{|l|}{ Lymphopenia, n [\%] } \\
\hline Normal ( $\geq 1,000 / \mu \mathrm{L})$ & $3[16]$ & 4 [29] & $5[45]$ & $1[20]$ \\
\hline Mild (<800/uL) & $5[26]$ & $0[0]$ & $1[9]$ & $2[40]$ \\
\hline Moderate $(<600 / \mu \mathrm{L})$ & 6 [32] & 6 [42] & $3[27]$ & $0[0]$ \\
\hline Severe $(<200 / \mu \mathrm{L})$ & $2[11]$ & $1[7]$ & $1[9]$ & $2[40]$ \\
\hline \multicolumn{5}{|l|}{ Thrombocytopenia, n [\%] } \\
\hline Normal ( $\geq 150 / \mu \mathrm{L})$ & $1[5]$ & 3 [21] & $3[27]$ & $0[0]$ \\
\hline Mild $(<75,000 / \mu \mathrm{L})$ & $2[11]$ & $2[14]$ & $0[0]$ & $1[20]$ \\
\hline Moderate $(<50,000 / \mu \mathrm{L})$ & $3[16]$ & $1[7]$ & $0[0]$ & $0[0]$ \\
\hline Severe $(<25,000 / \mu \mathrm{L})$ & 4 [21] & $1[7]$ & $1[9]$ & $2[40]$ \\
\hline Use of G-CSF beyond D30, n [\%] & $7[37]$ & 4 [29] & 1 [9] & $1[20]$ \\
\hline
\end{tabular}

$\mathrm{n}=$ patients with data available during the specified time period. 
Table S3 Summary of CAR-T cell toxicities and their management

\begin{tabular}{lcc}
\hline CAR-T cell toxicity and management & Number of patients & Percentage \\
\hline CRS & 12 & 63 \\
Grade 1 & 8 & 42 \\
Grade 2 & 3 & 16 \\
Grade 3 & 1 & 5 \\
ICANS & 14 & 74 \\
Grade 1 & 8 & 42 \\
Grade 2 & 3 & 16 \\
Grade 3 & 2 & 11 \\
Grade 4 & 1 & 5 \\
Neutropenic fever & 14 & 74 \\
Use of tocilizumab & 6 & 32 \\
Use of steroids & 3 & 16 \\
\hline
\end{tabular}

